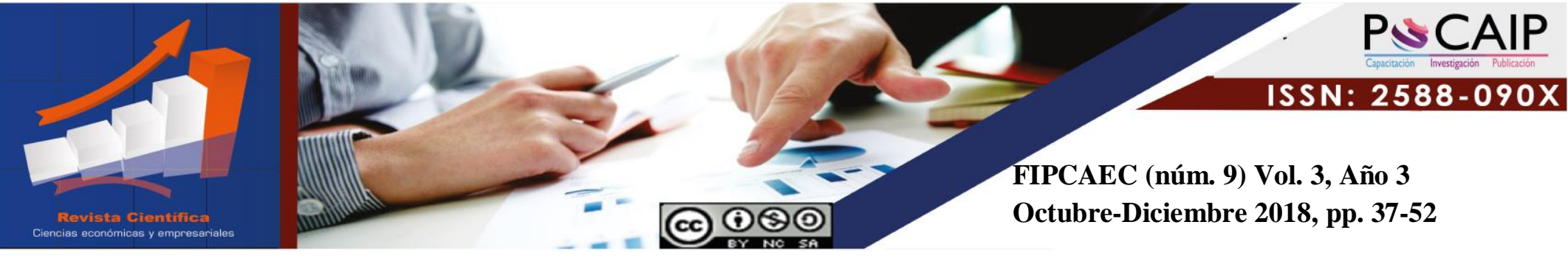

DOI: $10.23857 /$ fipcaec.v3i9.52

\title{
Evaluación del desempeño por competencias
}

\section{Performance evaluation by competencies}

\section{Avaliação de desempenho por competências}

\author{
Lenin Párraga-Zambrano ${ }^{1}$ \\ lenin_andres_p@hotmail.com \\ https://orcid.org/0000-0002-2070-6217
}

Correspondencia: lenin_andres_p@hotmail.com

* Recepción: 19/ 07/ 2018 *Aceptación: 05/09/ 2018 *Publicación: 15 /10/ 2018

Doctor en Ciencias Administrativas, Magíster en Gerencia Educativa, Diploma Superior en Liderazgo Educativo, Ingeniero Comercial, Docente de la Universidad Laica Eloy Alfaro de Manabí, Extensión Chone, Chone, Ecuador. 


\title{
Resumen
}

La investigación fue de carácter descriptivo con el objetivo de analizar el desempeño del talento humano de la cooperativa de suministros tecnológicos de Manabí. El 65\% de los encuestados no recibe comentarios referentes a su propio desempeño lo cual demuestra que la organización no es consciente del rol primordial que representan sus recursos humanos en desarrollo para fortalecer su desempeño, es decir que en dicha organización no se valora el recurso humano como ente proveedor que permite alcanzar los objetivos individuales relacionados directa o indirectamente con la organización. La evaluación del desempeño valora sistemáticamente el desempeño que posee cada persona en función de las actividades que cumple y de las metas y resultados a alcanzar ya que la misma debe realizarse siempre con proporción al perfil del puesto que ocupa para luego poder dar la retroalimentación y comentar los progresos del evaluado.

Palabras clave: Personal; personal profesional; trabajador; toma de decisiones.

\begin{abstract}
The research was descriptive in order to analyze the performance of the human talent of the technology supplies cooperative in Manabí. 65\% of respondents do not receive comments regarding their own performance which demonstrates that the organization is not aware of the primary role that its developing human resources represent in strengthening its performance, that is to say that said organization does not value human resources as a provider entity that allows to achieve the individual objectives directly or indirectly related to the organization. The performance evaluation systematically assesses the performance that each person possesses based on the activities they fulfill and the goals and results to be achieved since it must always be carried out in proportion to the profile of the position they occupy and then be able to give feedback and comment the progress of the evaluated.
\end{abstract}

keywords: Personnel; professional personnel; workers; decision making.

\section{Resumo}

A pesquisa foi descritiva, com o objetivo de analisar o desempenho do talento humano da cooperativa de suprimentos de tecnologia de Manabí. 65\% dos entrevistados não recebem 
comentários sobre seu próprio desempenho, o que demonstra que a organização não está ciente do papel principal que seus recursos humanos em desenvolvimento representam no fortalecimento de seu desempenho, ou seja, que essa organização não valoriza recursos humanos como uma entidade prestadora que permite alcançar os objetivos individuais diretamente ou indiretamente relacionados à organização. A avaliação de desempenho avalia sistematicamente o desempenho que cada pessoa possui com base nas atividades que cumpre e nos objetivos e resultados a serem alcançados, uma vez que deve sempre ser realizada na proporção do perfil da posição que ocupa e, em seguida, ser capaz de dar feedback e comentar o progresso dos avaliados.

Palavras-chave: Staff; equipe profissional; trabalhador tomada de decisão.

\section{Introducción}

En la evaluación de las competencias se develan fortalezas y debilidades que dan pie a prácticas de conocimiento como: compartir buenas estrategias, involucrar a la mayoría de la empresa en labores de inducción y entrenamiento, implementar el acompañamiento voluntario para quienes lo necesiten, convertir en tutor del más débil a quien posee el mejor desempeño en una competencia.

Hoy en día, los proyectos de investigación desarrollados por numerosas instituciones y las prácticas empresariales en el ámbito de los recursos humanos reflejan el enorme interés que existe por aplicar la gestión por competencias como una herramienta indispensable para profundizar en el desarrollo e involucramiento de su capital humano, el cual son las personas que ingresan, permanecen y participan en la organización, sin importar cuál sea su nivel jerárquico o su tarea, el recurso humano constituye el único recurso vivo y dinámico de la organización y es el que decide el manejo de los demás.

Evaluar el desempeño de un trabajador es una oportunidad para él, para la organización y para la comunidad de desempeñarse en una área específica donde sienta las comodidades para lograr su objetivo y el de la organización, es una oportunidad de ascenso y de crecimiento para la organización, por cuanto se puede mantener o mejorar la calidad del servicio que presta teniendo 
las personas claves e idóneas en los cargos que están ocupando y la comunidad consigue a su vez el beneficio de tener un servicio de calidad y que responde a las demandas de la misma de una manera satisfactoria, logrando así una mejor calidad de vida.

En la actualidad se reconoce que el éxito de las organizaciones está en la calidad del recurso humano que en esta labora, por lo que ha llevado a muchas organizaciones a invertir en la actualización y adiestramiento de estos recursos humanos en el área donde se encuentran desempeñando determinada tarea, evidenciándose la eficiencia y la eficacia en las tareas que deben cumplir, dejando como resultado una organización de calidad y que cumple con las exigencias demandadas por la sociedad y sus clientes, es decir, en esta actualización de conocimientos se ven beneficiados los trabajadores, la organización y la sociedad.

Es por ello que en las organizaciones se requiere del establecimiento de pautas, orientadas por competencias, así como de mecanismos auténticos de selección del personal para que ello garantice la incorporación de personal apto para cumplir con las tareas propias de cada cargo, posibilitándose elevar la eficiencia de la empresa, Aldana \& Piña (2017).

En este marco de ideas, se puede decir que la competencia es la construcción social de aprendizajes significativos y útiles para el desempeño constructivo en una situación real de trabajo que se obtiene no sólo a través de la instrucción, sino también en gran medida mediante el aprendizaje por experiencias en situaciones concretas de trabajo, posee competencia profesional quien dispone de los conocimientos, destrezas, aptitudes, necesarios para ejercer una profesión, puede resolver los problemas profesionales de forma autónoma, flexible, también está capacitado para colaborar en su entorno profesional y en la organización del trabajo., es decir, que la formación de competencias se da desde el desarrollo y fortalecimiento de habilidades de pensamiento complejo como clave para formar personas éticas, emprendedoras, competentes, logrando establecer una organización con un equipo fortalecido, visionario hacia el futuro de la misma, con objetivos, metas compartidas entre la organización y el personal humano que la conforma para obtener el éxito. 
De igual modo, para aprovechar un amplio marco de influencias de información sobre las competencias, es primordial que se maneje el término de recursos humanos, que no es una actividad nueva. Esta disciplina constituye el resultado histórico e integrado de la contribución de numerosos eventos y pioneros.

La función de administración de personal o administración de recursos humanos es definida por Werther \& Davis (1995):

Como aquellas prácticas que se requieren para llevar a cabo los aspectos relativos a las personas, pensando en el mejoramiento del desempeño y de las aportaciones del personal a la organización. La finalidad básica de la administración de personal es lograr que los trabajadores contribuyan a alcanzar las metas de la organización, mientras directa o indirectamente van a satisfacer sus necesidades individuales $o$ colectivas

Al existir la ausencia de evaluación de desempeño por competencias, la organización puede entrar en serias amenazas de fracaso, por cuanto, no se mide ni se evalúan las capacidades y las actitudes del recurso que se encuentra cumpliendo funciones laborales en determinada área, lo que puede llevar a un futuro incierto de la organización, debido a que posiblemente no cumpla con las metas que ésta tiene definida como empresa u organización, a su vez éste recurso puede no estar ubicado en el puesto donde tenga un mejor desempeño y rendimiento laboral, esto manifiesta la inadecuada gestión que desempeña el administrador del recurso humano.

Cabe destacar de lo antes expuesto, que la administración de recursos humanos busca mejorar las contribuciones productivas del personal a la organización, de manera que sean responsables desde un punto de vista estratégico, ético y social, tomando en cuenta en esta oportunidad la gestión por competencias, en ese sentido, la investigación tiene como objetivo analizar el desempeño del talento humano de la cooperativa de suministros tecnológicos de Manabí. 


\section{Desarrollo}

\section{Evaluación de desempeño por Competencia}

Es importante definir la definición de competencia laboral, en este sentido, Segura (2004) expone que:

La competencia es una capacidad laboral, medible, necesaria para realizar un trabajo eficazmente, es decir para producir los resultados deseados por la organización. Está conformada por conocimientos, habilidades, destrezas y comportamientos que los trabajadores deben demostrar y desarrollar para que la organización alcance sus metas y objetivos" y agrega que son: "capacidades humanas, susceptibles de ser medidas, que se necesitan para satisfacer con eficacia los niveles de rendimiento exigido en el trabajo (p. 4).

De allí que las competencias, es lo que hace que las personas tengan una dimensión de conductas abiertas y manifiestas, para realizar un trabajo o una actividad y lograr éxitos en la misma, lo que puede significar la conjunción de conocimientos, habilidades, disposiciones y conductas específicas. Si falta alguno de estos aspectos y el mismo hace falta para lograr algo, ya no es competente.

Por su parte, Ibarra (2001) la define como: "La capacidad productiva de un individuo que se define y mide en términos de desempeño en un determinado contexto laboral, y no solamente de conocimientos, habilidades o destrezas, sino incluyendo valores, rasgos y motivos que son la base del comportamiento" (p. 5). En efecto, las competencias poseen un carácter multidimensional, lo que las hace complejas, pues en ella están presentes características o aspectos relacionados a motivaciones, rasgos, formas de comportamiento, conocimientos, destrezas manuales y destrezas mentales y cognitivas, donde solo la forma de combinarse estos aspectos es lo que puede determinar cómo es el desempeño real del recurso humano.

La competencia se refiere pues, a la capacidad que muestra una persona de realizar una labor productiva, o decir que es propio de la persona hacer algo, lo hace con familiaridad, le resulta adecuado, fácil, lo que comprende, sabe lo que hace y por qué lo hace. A nivel mundial existen varias formas de entender las competencias laborales. Hellriegel y Slocum (2005, p. 16), indican las siguientes: 
1. Competencias Básicas: las competencias básicas se refieren a los capacidades elementales que posee un individuo, que le permiten adaptarse a los diferentes contextos, tanto laborales como de otra índole, como ejemplo se puede mencionar el poder comunicarse, de manera lógica para analizar y sintetizar hechos, esto enmarcado dentro de principios, valores y códigos éticos y morales.

2. Competencias Genéricas: las competencias genéricas o transversales se refieren a las capacidades requeridas en diversas áreas, sub áreas o sectores, que permiten llevar a cabo funciones laborales con niveles de complejidad, autonomía y variedad, similares. Las competencias genéricas están relacionadas con la capacidad de trabajar en equipo, de planear, programar, administrar y utilizar distintos recursos: tecnológicos, materiales, humanos, físicos, entre otros.

3. Competencias Específicas: son las capacidades laborales de índole específica de un área ocupacional o de competencia determinada, relacionadas con el uso de tecnologías y metodologías y lenguaje técnico para una determinada función productiva. Los tres tipos de competencia se conjugan para constituir la competencia integral del individuo. Por otro lado, Hellriegel y Slocum (2005), señalan que las competencias son un conjunto interrelacionado de habilidades, conductas, actitudes y conocimientos que necesita un individuo para ser eficaz en la mayoría de los puestos profesionales y gerenciales, definición que coincide con la de las competencias genéricas o transversales, e identifican siete competencias genéricas o básicas que consideran afectan en forma significativa el comportamiento en las organizaciones.

La evaluación de competencias no ha obtenido más que un éxito limitado en la medida en que se han hecho menos evaluaciones de lo que normalmente se realizaban. Hay que decir que la situación económica y el mercado laboral apenas favorecen el desarrollo de una iniciativa de este tipo. Se puede, en efecto, comprender que el empleado de una empresa dude al pedir una evaluación, ante el temor de ver interpretada su petición como un signo de debilidad.

En cambio, la evidencia de la importancia de las competencias en la evaluación de la experiencia profesional ha estimulado reflexiones y actividades que se inscriben en la evolución actual en la gestión de los recursos humanos. Más concretamente, análisis, ideas, y diligencias nuevas se han 
orientado hacia diversos temas de reflexión que conciernen a la definición de una nueva categoría de características individuales, las competencias, a la imagen que cada uno construye de sus competencias, a la posibilidad de identificarlas objetivamente y de desarrollarlas, así como a su importancia en las estrategias personales y de la empresa.

En este orden de ideas, Peña (2009, p. 57) expone que la aplicación de la evaluación del desempeño por competencias es importante para cualquier organización debido a que incluye los siguientes aspectos:

1. Mejoramiento del Rendimiento: la retroalimentación del rendimiento permite a los empleados, gerentes y especialistas de personal, intervenir con acciones apropiadas para mejorar el rendimiento.

2. Ajustes de Compensaciones: la evaluación del rendimiento ayudan a los encargados a tomar decisiones a determinar quién debe recibir aumentos de sueldo $\mathrm{y}$ sus bonificaciones, basándose en los méritos, lo que se determina primordialmente mediante evaluaciones de rendimiento.

3. Decisiones de Colocación: los ascensos, las transferencias y las degradaciones se suelen basar en el rendimiento del pasado o empleado. A menudo, los ascensos son una recompensa por el rendimiento del pasado.

4. Necesidades de Capacitación y Desarrollo: un mal empleado puede indicar una necesidad de capacitación. De modo similar, el buen desempeño puede indicar potencial desaprovechamiento que se debería desarrollar.

5. Planeación y Desarrollo de Carreras: la retroalimentación sobre el desempeño dirige las decisiones de carrera sobre las proyectarías específicas que deben investigarse.

6. Inexactitudes de la Información: un mal desempeño puede indicar errores en la información de análisis de empleo, los planes de recursos humanos u otras partes del sistema de información de análisis de empleo, los planes de recursos humanos u otras partes del sistema de información de la administración del personal. La dependencia de información inadecuada puede haber producido decisiones incorrectas de contratación, adiestramiento o asesoramiento.

7. Errores del Diseño de Empleos: un mal desempeño puede ser síntoma de diseños de empleos mal concebidos. La evaluación contribuye a diagnosticar esos errores. 
8. Igualdad de Oportunidades de Empleo: las evaluaciones precisas del desempeño que miden en calidad el rendimiento relacionado con los empleos aseguran que las decisiones internas de colocación no son discriminatorias.

9. Desafíos Externos: a veces, el desempeño se ve afectado por factores situados fuera del ambiente de trabajo, tales como asuntos familiares, financieros, de salud o personales, o de otros tipos. Si se descubren por medio de evaluaciones, el departamento de personal puede proporcionar ayuda.

Cualquier previsión de la evaluación del rendimiento del grupo debe empezar por reconocer que los grupos de trabajo forman parte de una organización mayor y que los factores, como la estrategia de la organización, la estructura de autoridad, los procedimientos de selección y el sistema de recompensas, puede propiciar un clima favorable o desfavorable para el funcionamiento del grupo.

Ante esta circunstancia, la evaluación de desempeño por competencia surge como una de las técnicas para la administración eficiente del desempeño del recurso humano. La evaluación por competencias, es un proceso complejo que requiere pasos previos entre ellos la definición de perfiles ocupacionales estructurados en torno a conocimientos, habilidades, conductas individuales y sociales. Por ello es necesario establecer los instrumentos de medición que den cuenta de las demostraciones o evidencias de cada una de estas competencias, pero vistas desde una perspectiva balanceada e integral, la cual permitirá detectar las falencias y carencias en las personas que ocupan determinados puestos de trabajo, y las cuales podrán ser sujetas a un proceso de capacitación posterior. De manera tal, que esta herramienta aborda un aspecto indispensable para el manejo integral de los recursos humanos en todo tipo de organización.

Según Garza (2005) desde el punto de vista organizacional, "la evaluación del desempeño por competencias es un modelo que permite alinear el capital intelectual de una organización con su estrategia de negocios, y facilitar al mismo tiempo el desarrollo profesional de las personas" (p. 807). Mientras que desde el punto de vista personal, se convierte en una revolución en el sentido tradicional de calidad, pues se considera que: Para ser un buen profesional se requiere tener unas 
cualidades mínimas como persona, las cuales deben ser tomadas en cuenta dentro del sistema de evaluación de desempeño de cualquier organización.

De allí que, tomando en consideración las condiciones básicas del proceso de medir el desempeño por competencias, sea necesario esbozar un instrumento que recoja la impresión tanto del tipo de persona como del tipo de profesional que labora en la organización, que abarque la mayor cantidad de datos que aseguren la fiabilidad de la información que se requiere saber y con la cual se orientará la toma de decisiones. La evaluación del desempeño por competencias es un proceso que se diseña siguiendo las características, intereses y necesidades propias de cada organización, cuyo proceso puede convertir a los recursos humanos en recursos estratégicos, al permitir diferenciar la organización de otras.

De lo expuesto se tiene que para el diseño de un sistema de evaluación del desempeño por competencias este tiene que considerarse como un proceso como de medición, acompañamiento y ajuste permanente de los procesos laborales, por lo que se estudian las competencias las cuales se deben comunicar, comprender mediante la coevaluación y autoevaluación que permita al personal en sus acciones conductuales, laborales, emocionales e intelectuales.

\section{Condiciones Básicas para la evaluación por Competencias}

Es de indicar que para alcanzar éste objetivo básico la organización debe intentar alcanzar tanto los objetivos fundamentales, como ciertos objetivos intermedios. De allí que es importante indicar las condiciones básicas de todo proceso de evaluación de competencias laborales, tal como lo indica Garza (2005, p. 810) son las que se refieren a su:

1. Validez: Debe responder a la pregunta, ¿puede el instrumento que voy a aplicar captar realmente si la persona es competente o no? Por ejemplo, que es mejor observar al evaluado en su puesto de trabajo o en su proceso de simulación.

2. Autenticidad: Establecer con certeza que el evaluado es quien desarrollo tal o cual demostración o prueba. El punto es importante cuando se trata de evaluar el trabajo de un fotógrafo, por ejemplo, que aporta como prueba algunas fotografías. ¿Cómo se certifica que el autor de las muestras es efectivamente el evaluado? 
3. Suficiencia: ¿Cuántas veces debemos observar un proceso para estar seguros de que el evaluado efectivamente demuestra la competencia que estamos evaluando? El principio a aplicar en este caso es que el sector productivo y el consejo de la unidad administrador del sistema de acreditación de competencias laborales definen la cantidad de veces exigidas y la cantidad de experiencia necesaria para ser considerado competente.

4. Justicia: Donde todas las personas que aspiran al reconocimiento de sus competencias laborales son evaluadas con estándares, criterios e instrumentos similares.

5. Aceptación: Donde las personas que se van a someter al proceso de evaluación de competencias laborales para un nivel $\mathrm{X}$, aceptan y reconocen los estándares, criterios e instrumentos de evaluación aplicables son los correctos y justos, naturalmente esta aceptación se extiende a los empresarios y a quien contratan en general.

Todas estas funciones de la evaluación del rendimiento son importantes, aunque su importancia depende de la posición que se adopte. Algunas son claramente importantes para las decisiones de administración de personal. Sin embargo, el interés está depositado en el comportamiento organizacional. Por tanto, se está resaltando la evaluación del desempeño en su papel de mecanismo para ofrecer retroalimentación y como determinante de la distribución de recompensas.

\section{Metodología de la investigación}

La investigación fue de carácter descriptivo, Hernández, Fernández y Batista (2014) la define como aquella que "busca especificar propiedades, características y rasgos importantes de cualquier fenómeno que se analice", así mismo indican que la investigación no experimental "son estudios que se realizan sin la manipulación deliberada de variables y en los que solo se observan los fenómenos en su ambiente natural para después analizarlos”.

La población que se estudió estuvo conformada por setenta y siete (77) personas que laboran en la cooperativa de suministros tecnológicos de Manabí, aplicándose un instrumento dicotómico de 37 ítems, obteniéndose un coeficiente de Kuder de Richardson de 0,89 catalogándose como altamente confiable para su aplicabilidad. 


\section{Resultados}

\section{Cuadro 1}

Proceso de evaluación

\begin{tabular}{lll} 
Alternativas & Frecuencia & Porcentaje \\
\hline SI & 33 & 43 \\
NO & 44 & 57 \\
TOTAL & 77 & 100 \\
\hline
\end{tabular}

Lo arrojado por la muestra evidencia que la mayoría de los encuestados (57 \%) no están de acuerdo con que el proceso de evaluación sea justo y objetivo, esto debido a que no se aplican procedimientos dirigidos para ampliar, y estimular los conocimientos y destrezas del personal .

\section{Cuadro 2}

Tipo de evaluación

\begin{tabular}{lll} 
Alternativas & Frecuencia & Porcentaje \\
\hline SI & 51 & 66 \\
NO & 26 & 34 \\
TOTAL & 77 & 100 \\
\hline
\end{tabular}

Como se puede observar en el cuadro $\left(\mathrm{N}^{\circ} 2\right.$ nos arroja que un $66 \%$ si han tenido algún motivo para cambiar la evaluación que le diera a alguien de su personal, esto debido a que parte del personal carece de un grado de instrucción acorde al cargo que ocupa. A lo que los supervisores han brindado poco interés, pues a los empleados no se les han realizado programas de planificación de carrera o no se les ha incentivado a realizar estudios para que pudiesen tener un nivel académico de acuerdo a sus funciones. 


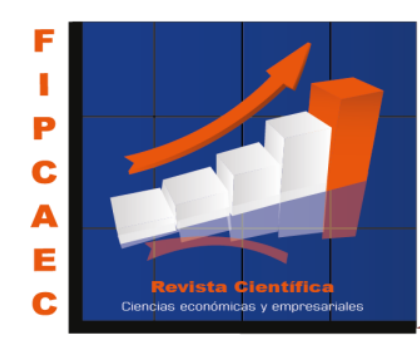

\section{Cuadro 3}

Autoevaluación

\begin{tabular}{lll} 
Alternativas & Frecuencia & Porcentaje \\
\hline SI & 27 & 35 \\
NO & 50 & 65 \\
TOTAL & 77 & 100 \\
\hline
\end{tabular}

Los resultados obtenidos en este ítem muestran que un buen porcentaje de los encuestados (65\%) no recibe comentarios referentes a su propio desempeño lo cual demuestra que la organización no es consciente del rol primordial que representan sus recursos humanos en desarrollo para fortalecer su desempeño, es decir que en dicha organización no se valora el recurso humano como ente proveedor que permite alcanzar los objetivos individuales relacionados directa o indirectamente con la organización.

\section{Cuadro 4}

Entrenamiento gerencial

\begin{tabular}{lll} 
Alternativas & Frecuencia & Porcentaje \\
\hline SI & 17 & 22 \\
NO & 60 & 78 \\
TOTAL & 77 & 100 \\
\hline
\end{tabular}

Lo arrojado por la muestra evidencia que la gran mayoría de los encuestados (78\%) afirman que existe deficiencia en cuanto a si se aplican procedimientos dirigidos para ampliar, y estimular entrenamiento en materias de supervisión o gerencia para el personal administrativo de la organización lo cual demuestra que es una desventaja para la organización por cuanto se verían afectadas las capacidades, habilidades y destrezas que posean los miembros de la misma 


\section{Conclusiones}

La evaluación del desempeño valora sistemáticamente el desempeño que posee cada persona en función de las actividades que cumple y de las metas y resultados a alcanzar ya que la misma debe realizarse siempre con proporción al perfil del puesto que ocupa para luego poder dar la retroalimentación y comentar los progresos del evaluado.

Por su parte, las competencias buscan ver los comportamientos, características y habilidades medibles que definen a los trabajadores y le permiten tener un desempeño superior al esperado en su puesto de trabajo logrando que la organización en la que labora alcance sus metas y objetivos.

Los resultados arrojados en los cuestionarios señalaron, según las tabulaciones pertinentes que el personal de apoyo administrativo tiene tiempo laborando dentro de la institución y en el cargo que ocupan actualmente, como para conocer la filosofía, misión, visión de la organización y las labores inherentes a su cargo por su experiencia razón de peso para afirmar que este personal merece la aplicación de un sistema que cause incidencia positiva en ellos, generando beneficios que puedan satisfacer sus necesidades y expectativas en su crecimiento profesional y personal.

\section{Referencias}

1. Aldana, J., \& Piña, J. (2017). Calidad del servicio prestado al cliente por los instructores de gimnasios. Revista Arbitrada Interdisciplinaria Koinonía, 2(3), 172-197. Recuperado de http://fundacionkoinonia.com.ve/ojs/index.php/revistakoinonia/article/view/59/46

2. Garza J. (2005) Valores para el Ejercicio Profesional. McGraw Hill. Mexico.

3. Hernández, R., Fernández, C., Baptista, P. (2014). Metodología de la Investigación. McGraw Hill. Colombia.

4. Hellriegel, D., Jackson, S. \& Slocum, J. (2005) Administración, un enfoque basado en competencias (10 ad. Ed.) México: Thomson

5. Ibarra, A. (2000). Formacion de recursos humanos y competencia laboral. Voletin Cinterfor Monte video. 


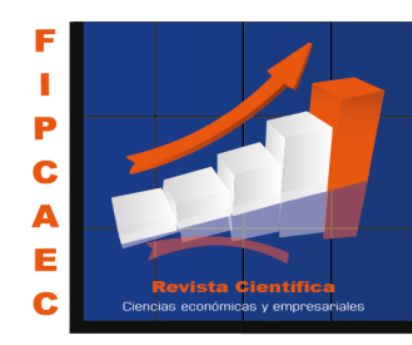

6. Peña, C. (2009). Evaluación del desempeño del Talento Humano. Disponible en: http://gerenciacarlos.zoomblog.com/archivo/2009/10/25/evaluacion-del-desempeno-deltalento-h.html

7. Segura, L. (2004) Gestión del Recurso Humano basada en competencias laborales. ( $2^{\mathrm{a}}$. Ed.). Guatemala: Impresos Colindres.

8. Werther, W. \& Davis, K. (2000). Administración de Personal y Recursos Humanos. México: Mc Graw Hill.

\section{References}

1. Aldana, J., \& Piña, J. (2017). Quality of service provided to the client by gym instructors. Interdisciplinary Arbitrated Review Koinonía, 2 (3), 172-197. Recovered from http://fundacionkoinonia.com.ve/ojs/index.php/revistakoinonia/article/view/59/46

2. Garza J. (2005) Values for Professional Practice. McGraw Hill Mexico.

3. Hernández, R., Fernández, C., Baptista, P. (2014). Investigation methodology. McGraw Hill Colombia.

4. Hellriegel, D., Jackson, S. \& Slocum, J. (2005) Administration, a competency-based approach (10th Ed.) Mexico: Thomson

5. Ibarra, A. (2000). Training of human resources and labor competence. Vointer Cinterfor Monte video.

6. Peña, C. (2009). Evaluation of the performance of Human Talent. Available at: http://gerenciacarlos.zoomblog.com/archivo/2009/10/25/evaluacion-del-desempeno-deltalento-h.html

7. Segura, L. (2004) Human Resource Management based on job skills. (2nd ed.). Guatemala: Printed Colindres. 
8. Werther, W. \& Davis, K. (2000). Personnel management and human resources. Mexico: Mc Graw Hill.

(C2018 por el autor. Este artículo es de acceso abierto y distribuido según los términos y condiciones de la licencia Creative Commons Atribución-NoComercial-Compartirlgual 4.0 Internacional (CC BY-NC-SA 4.0) (https://creativecommons.org/licenses/by-nc-sa/4.0/). 